\title{
Inhalt des 3. Jahrganges
}

L. von Brunn, Hippokrates und die meteorologische Medizin 151

H.Bueß, Briefe Laurenz Sondereggers an Arnold Baader 193

Hans Debrunner, Michel de Montaigne und die Lehre von der Mißbildung 1

Hubert Erhard, Die Entdeckung der Parthenogenesis durch Charles Bonnet 15

Hans Fischer, Ein unveröffentlichter Brief Conrad Geßners (1516-1565) an Johannes Fabricius Montanus in Chur 125

H.M.Fraser, Moufet's Theatrum Insectorum 131

O.Gigon, Die naturphilosophischen Voraussetzungen der antiken Biologie 35

P. Jung, Die Augsburger erneuerte Hebammenordnung von $1750 \quad 134$.

B. Milt, Schweizerische Theophrastforschung und schweizerische Theophrast-
editionen im 16. Jahrhundert und ihre Bedeutung

Bernhard Milt, Some explanatory notes to M. H. M. Fraser's Article about Moufet's Theatrum Insectorum 132

- Johannes Geßner (1709-1790) 103

E.Moser, Dr.med. Johann Melchior Aepli aus Dießenhofen 174

Henry Nigst, Zur Frage der Entdeckung und Benennung der Glandulae $\begin{array}{lr}\text { duodenales } & 8\end{array}$

B.Peyer, Über die zoologischen Schriften des Aristoteles 58

Gustav Senn, Liste der Veröffentlichungen G.S. zur Geschichte der Natur$\begin{array}{ll}\text { wissenschaften, hauptsächlich der Antike } & 97\end{array}$

\section{Buchbesprechungen}

Barclay, Franklin, Prichard, The foetal circulation (H. Fischer) 213

In Memoriam Gustav Senn, Basel 1945 (H. Fischer) 96

Douglas Guthrie, A History of Medicine (H. Fischer) 148

Journal of the History of Medicine and allied Sciences. A Quarterly. New York 1946 (H. Fischer) 149

C. G.Jung, Psychologie und Alchemie (B. Milt) 30

Nova Acta Paracelsica, II. Jahrbuch d. schweiz. Paracelsus-Gesellschaft, 1945 (B. Milt)

Reinhold Strömberg, Theophrastea (B. Milt) 27

- - Griechische Pflanzennamen (B. Milt) 93 